Itinéraires Itinéraires

Littérature, textes, cultures

2020-2 | 2020

Discours animal. Langages, interactions, représentations

\title{
Écouter les animaux parler. Présentation du numéro
}

Listening to Animals Talking. Presentation of the Issue

Laura Goudet, Marie-Anne Paveau et Catherine Ruchon

\section{OpenEdition}

\section{Journals}

Édition électronique

URL : https://journals.openedition.org/itineraires/8756

DOI : $10.4000 /$ itineraires. 8756

ISSN : 2427-920X

Éditeur

Pléiade

Référence électronique

Laura Goudet, Marie-Anne Paveau et Catherine Ruchon, «Écouter les animaux parler. Présentation du numéro », Itinéraires [En ligne], 2020-2 | 2020, mis en ligne le 06 décembre 2021, consulté le 06

décembre 2021. URL : http://journals.openedition.org/itineraires/8756 ; DOI : https://doi.org/10.4000/ itineraires.8756

Ce document a été généré automatiquement le 6 décembre 2021

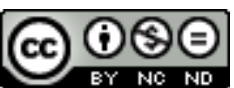

Itinéraires est mis à disposition selon les termes de la licence Creative Commons Attribution - Pas d'Utilisation Commerciale - Pas de Modification 4.0 International. 


\section{Écouter les animaux parler. Présentation du numéro}

Listening to Animals Talking. Presentation of the Issue

Laura Goudet, Marie-Anne Paveau et Catherine Ruchon

\section{Cadres et objectifs épistémologiques du numéro}

Depuis quelques années, on commence à percevoir des formes de langage animal par le filtre de différentes disciplines, qui vont de la zoologie et de l'anthropologie jusqu'à l'éthologie ou la zoosémiotique. Cette interdisciplinarité renforcée par les courants animalistes et les débats sur le droit animal fait bouger les frontières spécistes, redonnant à l'humain une place d'hyponyme. L'opposition humain-animal s'estompe et l'on parle aujourd'hui des " autres animaux ${ }^{1}$ ». Autour de ce numéro se sont rassemblés des chercheure's qui donnent la parole aux animaux en tentant de prendre leur point de vue. Il s'agit de linguistes, mais aussi de sémioticien'ne's, de philosophes ou encore de spécialistes de littérature, qui ensemble ouvrent la voie d'une linguistique au-delà de l'humain.

\subsection{La question animale : questionnement de l'évidence spéciste}

2 À la fin du XVIII ${ }^{\mathrm{e}}$ siècle, les animaux ont commencé à disparaître des sociétés dites occidentales. Dans « Pourquoi regarder les animaux? », John Berger explique comment l'industrialisation, touchant tous les domaines de ces sociétés (travail, transports, nourriture, loisirs) a progressivement marginalisé les animaux jusqu'à les faire disparaitre de la vie quotidienne des humainees (Berger [1995] 2010). Il souligne un symptôme paradoxal de cette disparition: la multiplication des représentations animales, notamment anthropomorphiques, en particulier dans les produits culturels de l'enfance, mais également dans la publicité ou les discours pédagogiques. L'anthropomorphisme, qui faisait selon lui "partie intégrante de la relation entre l'homme et l'animal [...], expression de leur proximité [et] trace d'un usage 
ininterrompu de la métaphore animale », est ainsi devenu objet de malaise et de gêne (Berger [1995] 2010 : 37-38).

3 Ces trente dernières années, la question animale s'est imposée dans l'espace public mais également dans la recherche en sciences humaines et sociales, questionnant la doxa spéciste sur laquelle reposent bien des fondements de ces mêmes sociétés (Calarco 2010). D'abord à bas bruit, puis plus visiblement ces dix dernières années, militant·e·s, intellectuel-le's et chercheure's se sont mis·e's à interroger la séparation des ordres humain et animal, et les termes spécisme et antispécisme, animalisme, véganisme, droit des animaux, ou (proto)morale animale se sont imposés dans les discours profanes, médiatiques et scientifiques. Le spécisme se définit comme une distinction hiérarchisée de l'ordre humain et de l'ordre animal, le second étant considéré comme inférieur par les manques de ce qui ferait l'«exception humaine» (Schaeffer 2007): aptitude à l'émotion, expérience de la douleur pour certaines espèces, faculté de communication et/ou de langage, présence d'une forme de conscience (sentience animale). Cette distinction radicale entre l'humaine et l'animal a des fondements philosophiques connus (notamment à partir de Descartes), mais également économiques et politiques. L'idéologie spéciste, si peu questionnée qu'elle tient lieu d'évidence, a en effet soutenu la relégation et l'exploitation des animaux, considérés légalement comme des objets, comme outils d'élaboration et de maintien de la supériorité humaine, entraînant la domination de l'humaine sur l'ensemble de la planète, domination qui n'est d'ailleurs pas sans lien avec d'autres paradigmes d'oppression comme le racisme et le sexisme (Rivera 2015).

\subsection{Antispécisme, postdualisme et perspective écologique : cultures et pensées animales}

4 Le cadre de ce numéro, qui porte sur la question du langage, du texte et de la représentation, est non spéciste, ce qui implique une approche indissociablement épistémologique, politique et théorique qui s'écarte de l'anthropocentrisme (idée que la référence du langage et de la pensée est seulement humaine) et du logocentrisme (idée que les langages et les textes ne sont analysables qu'à partir d'eux-mêmes) et intègre une composante politique à la pratique scientifique (Paveau 2012a, 2017). Nous nous inscrivons dans une pensée non dualiste et écologique à la suite des travaux de Latour (1991, 1995, 1999), Descola $(2006,2011,2014)$ ou Kohn ([2013] 2017), pour ne citer qu'eux, qui ont patiemment défait l'édifice de la pensée dualiste et en particulier l'opposition binaire entre nature et culture. Cela implique, dans le travail sur les animaux, de prendre acte d'une « rupture de nos encadrements conceptuels » (Calarco 2010 : 85) proposée par le précurseur Uexküll (1934), puis, entre autres Derrida (1989, $1994,2006)$, Lestel $(2001,2010)$, Fontenay $(1998,2008)$, pour envisager les animaux dans leurs liens, leurs proximités, voire leur identité avec les humaineess, et non leurs distinctions ou leurs manques par rapport à ce qui serait une perfection humaine ; cela en évitant la tentation anthropocentrique (voire carnophallogocentrique, selon le mot de Derrida 1989), qui est jusqu'à présent une des manières les plus fréquentes de doter l'animal d'une vie propre et d'une intelligence.

5 Cette approche repose sur un nombre important de travaux qui, depuis une cinquantaine d'années, ont montré que les animaux possédaient des cultures, des représentations internes, des valeurs morales et des facultés, si ce n'est langagières, du 
moins protolangières et en toute certitude sémiotiques et communicationnelles. Il existe en effet des cultures animales, puisque les animaux sont capables d'apprendre et de transmettre. La notion de culture animale, formulée très tôt par Jakob von Uexküll sous le nom de «monde propre» (von Uexküll 1934), est développée par Dominique Lestel dans Les origines animales de la culture, publié en 2001 et devenu un classique, et vulgarisée par Frans de Waal dans plusieurs ouvrages grand public (de Waal 2001). Sujets culturels, les animaux sont également des sujets cognitifs: ils trompent (Premack et Premack 1984 [1983]), portent des ornements (de Waal 1996) et sont dotés d'états mentaux (Proust 1997, 2000, 2010). On peut aussi les considérer comme des sujets moraux : ils sont altruistes et connaissent l'empathie (de Waal 1996, 2010), ce qui les dote d'une morale certes naïve mais réelle (Nurock 2008), liée à leur organisation sociale (Depenau 2009). L'ensemble de ces capacités fait des animaux de véritables actants dans le monde, ce qui nous oblige, selon l'historien Éric Baratay, à les intégrer comme paramètre dans les recherches en SHS, au même titre que tous les autres actants (Baratay 2017).

\section{L'hypothèse d'un discours animal}

6 Les questions traitées dans ce numéro découlent de ces acquis, permettant l'exploration de ce que nous avons décidé d'appeler «discours animal». Cette hypothèse implique d'avoir une position sur l'anthropomorphisme, d'admettre un décentrement du langage et de défendre l'idée d'un protolangage animal.

\subsection{La question de l'anthropomorphisme}

7 Par discours animal, nous entendons bien un discours qui est produit par les animaux, d'une manière non métaphorique ; nous pensons, dans la lignée des travaux cités plus haut, et à partir de positions philosophiques et politiques, que les animaux parlent, et que nous devons leur donner une voix, "sans que celle-ci ne s'exprime à travers des humains ventriloques" (Descola 2017: 12). Certain'es linguistes trouvent que l'hypothèse d'un discours animal est difficilement tenable, et même "bizarre ", la faculté de langage étant pour elleux exclusivement humaine, et la parole des animaux ne pouvant être qu'une fiction anthropomorphique. Quand illes se saisissent de la thématique animale, qui commence à être à la mode dans les lieux de recherche actuellement, les linguistes restent en général anthropocentrée's et logocentrée'es, travaillant essentiellement, et entre guillemets, sur la manière dont les textes représentent ou décrivent les animaux, souvent à travers les textes littéraires (voir par exemple Rabatel 2017). Ce faisant, illes témoignent d'une méfiance envers l'anthropomorphisme bien installée dans la littérature sur le langage animal, et que représente bien cette remarque de Trevor Harley à propos du célèbre cas d'Alex, le perroquet gris du Gabon étudié par Irene Pepperberg pendant une trentaine d'années (Pepperberg 2006, 2009): "Il faut faire attention à l'anthropomorphisme [...]. Pepperberg connaissait ce danger potentiel et a documenté précautionneusement ses recherches sur Alex » (Harley 2017 : 38). La question du langage animal est en effet controversée et le "risque » de l'anthropomorphisme est l'un des arguments majeurs de la contestation d'une véritable activité langagière de l'animal. Mais on peut penser, comme le soulignait John Berger, et comme le pense Frans de Waal, qui lui oppose l'« anthropodéni » (un déficit d'anthropomorphisme) que l'anthropomorphisme est au 
contraire un signe de décentrement et un outil non spéciste et écologique nécessaire pour envisager une subjectivité animale (de Waal 2001, 2016).

\subsection{Le décentrement du langage}

8 À partir du point de vue non dualiste (vs anthropocentré) et écologique (vs logocentré) qui est le nôtre, la question pour nous n'est plus de savoir si les animaux parlent ou non, mais comment ils le font. Nous savons, depuis la fameuse controverse entre Benveniste et Fritsch à propos du «langage» des abeilles (Benveniste 1952) que les animaux ne produisent pas de double articulation du langage et qu'on ne peut parler spécifiquement de langage et de langue concernant la communication animale (Mondémé 2018). Mais, à la suite de Fernando Kohn, lui-même s'inscrivant dans la sémiotique peircienne, nous considérons que la conception de la représentation en sciences du langage est trop étroite (le signe y est un symbole, c'est-à-dire une forme liée de manière arbitraire à une chose représentée, qui doit faire l'objet d'un apprentissage) et nous lui préférons une conception plus large, qui y fait entrer l'icône et l'indice. Pour Eduardo Kohn, il est nécessaire de "défamiliariser le signe conventionnel en montrant qu'il ne constitue qu'une seule des différentes modalités sémiotiques, puis d'examiner les différentes propriétés non symboliques de ces autres formes sémiotiques qui sont généralement occultées par le symbolique ou qui lui sont assimilées dans l'analyse anthropologique " ([2013] 2017 : 39). Autrement dit, au signe saussurien (le «signe conventionnel»), relevant stricto sensu de la règle arbitraire et apprise, nous préférons le signe peircien («différentes modalités sémiotiques»), qui fait parler plus directement et concrètement les images et les traces. À l'instar d'Eduardo Kohn, nous souhaitons " provincialiser le langage ", c'est-à-dire le décentrer de son arrimage symbolique et logocentré pour envisager des manières plurielles de signifier, à partir d'une conception du signe plus large, qui puisse englober les êtres et les objets non humains (Paveau 2012b).

9 L'hypothèse d'un discours animal est inédite en linguistique et analyse du discours tant en France qu'à l'étranger, la question étant surtout traitée en sciences cognitives et en éthologie. Un groupe de chercheuree's en sémiotique, l'équipe GASP de Paris 8 dirigée par Denis Bertrand et Michel Costantini, a lancé récemment un projet relevant de la zoosémiotique, en posant la question de l'énonciation animale (Bertrand et Costantini 2017). Celle-ci est conçue comme une émission de significations de la part des animaux, plutôt de l'ordre de la sémiose que de l'énonciation véritable, en tout cas hors de l'ordre du langage proprement dit. Gianfranco Marrone explique par exemple qu'il s'agit de renouveler la première zoosémiotique (la «zoosémiotique $1.0 »)$, qui s'interrogeait sur les capacités langagières et communicationnelle des animaux dans les années 1960 et 1970, pour ouvrir une "zoosémiotique 2.0 », qui analyserait « comment ils sont parlés à l'intérieur de discours sociaux, et, parallèlement, comment ils deviennent sujets et objets de toutes les activités pragmatiques cognitives, passionnelles et sensorielles présentes dans les différentes sociétés " (Marrone 2018: 10). Les travaux, menés dans une perspective qui semble postdualiste et non spéciste, sont cependant majoritairement menés à partir de l'énonciation humaine, sur des corpus littéraires notamment, avec l'idée de donner une place aux animaux dans un collectif hybride plus qu'une voix véritable leur reconnaissant une subjectivité propre. 


\subsection{Productions (proto)langagières animales}

10 Notre perspective est différente pour deux raisons : d'abord, la linguistique et l'analyse du discours n'ont jamais envisagé un langage ou une parole animale, restant, si l'on peut dire, désespérément spécistes, et l'idée de prendre au sérieux une parole animale est donc tout à fait nouvelle; ensuite notre approche comporte une dimension politique qui nous intime d'accorder aux animaux une sentience, une voix, une subjectivité et des droits, autrement dit de les considérer comme des sujets; ils sont donc pour nous des sujets de discours. Nous postulons, avec ces orientations et à partir d'un corpus de recherches important, menées en éthologie, biologie animale, zoologie, anthropologie animale ou zoosémiotique «1.0», qui ont posé depuis longtemps l'existence d'une production de signes par les animaux (pour une synthèse voir Vauclair 1995), que les animaux produisent des énoncés relevant de la représentation, et des messages relevant de la communication intraspécifique et interspécifique. Nous travaillons donc principalement la parole des animaux à partir d'eux-mêmes et de leurs "provinces » et non à partir de la manière dont l'humaine se posant en "centre " légitime, leur «prêterait » une voix que, finalement, dans un spécisme résistant, ille leur dénierait (Goudet, Paveau et Ruchon 2017).

\section{Présentation des articles}

La participation au numéro avait été ouverte aux propositions émanant de toutes les disciplines à condition qu'elles portent sur les langages, les textes, les signes ou les discours animaux, en français ou en anglais, et qu'elles montrent que la prise en compte des productions animales déplace les frontières épistémiques dualistes et spécistes habituellement posées en lettres, sciences du langage et sciences humaines et sociales.

Le numéro était également ouvert aux discours animaux imaginaires (anthropomorphismes), en littérature notamment, puisqu'il existe des travaux importants sur cette question, fédérés notamment dans le projet ANR de zoopoétique Animots dirigé par Anne Simon, consacré à la recherche sur les bêtes et l'animalité dans la littérature des $\mathrm{xx}^{\mathrm{e}}$ et $\mathrm{xxI}^{\mathrm{e}}$ siècles (on en trouvera une synthèse dans Benhaïm et Simon dir. 2017, Simon 2015, et sur le carnet du projet:https:// animots.hypotheses.org/).

Les propositions que nous avons sélectionnées et qui constituent le numéro ressortissent à trois domaines bien identifiés : la représentation littéraire des paroles animales, la réflexion linguistique et cognitive sur une faculté de parler animale et la question de l'anthropomorphisme.

La première partie, intitulée, "Paroles animales en littérature ", est consacrée à la perspective littéraire sur la faculté de parler animale et rassemble quatre articles. Dans " "Le rossignol trouvera bon de parler lui-même de soi-même." Discours animal et subversion libertine dans les États et Empire du Soleil de Cyrano de Bergerac ", Aude Volpilhac montre que les oiseaux parleurs sont loin de n'être qu'une figure chez Cyrano, mais constituent une véritable proposition philosophique, celle d'une remise en cause de la hiérarchie des êtres. L'article d'Élisabeth Plas, intitulé «"(Ainsi parlent les araignées)". Les prosopopées sans anthropocentrisme de l'histoire naturelle romantique ", étudie la manière dont Jules Michelet, dans son cycle naturaliste 
(L'oiseau, L'insecte, La mer, La montagne), élabore un anthropomorphisme singulier, qui évite la tentation de parler à la place des animaux, en créant une expressivité animale qu'on pourrait dire située, c'est-à-dire essayant de respecter le point de vue des bêtes. C'est aussi la question de l'expressivité animale qui occupe Sophie Milcent-Lawson, dans "Imaginaires zoolinguistiques: des langues animales dans la fiction littéraire »: sous un autre angle, celui de la linguistique, elle rend compte de la grammaire de ces véritables " uglossies » créées en littérature au cours des siècles. Cette description des langages animaux en contexte littéraire s'achève par une exploration des langages spécifiques aux chiens menée par Maria do Rosário Girao Ribeiro Dos Santos, dans "Chiens sentients: du langage émotif au discours critique", à travers quatre œuvres de Virginia Woolf, Paul Auster, Manuel Alegre et Emilio Ortiz.

Cette entrée sur le langage animal dans l'univers littéraire se prolonge par l'étude de la pensée animale et de la faculté de langage animal au travers de différentes disciplines, neurologie, éthologie cognitive, linguistique. Intitulée «D'une faculté de langage des animaux ", cette seconde partie s'ouvre par une réflexion philosophique sur la capacité langagière des animaux qui s'appuie sur les résultats des neurosciences et de l'éthologie cognitive. Dans "Sapiens Animalis. La pensée spécifie-t-elle l'humain?", Mouhamadou El Hady Ba remet en question le caractère opératoire d'une distinction entre animal humain et animal non humain sur la base des catégories de la cognition, voire de la conscience. En revanche, l'auteur démontre de façon convaincante par de nombreux exemples que les animaux non humains peuvent faire preuve d'intentionnalité et ne sont pas dénués de représentations mentales. Cet article met en lumière un courant de pensée réfutant l'exception humaine, qui s'appuie sur une réflexion ancienne, notamment chez les humanistes, mais qui trouve encore aujourd'hui peu d'applications dans les sciences « humaines ", tout particulièrement en linguistique. C'est ce que démontre l'article de Marie-Anne Paveau et de Catherine Ruchon, «La linguistique et le langage animal. Résistances, décentrements, propositions ", au travers d'un parcours historique et épistémique de cette "exception linguistique ». Là aussi se pose le problème de définitions inadaptées puisque élaborées à partir du repère humain, avec en premier lieu le principe de double articulation du langage. Peut-être parce qu'elles ne sont pas sous l'emprise de dogmes théoriques, d'autres disciplines parviennent à mettre en valeur les spécificités d'un langage animal. C'est le cas de l'éthologie, mais aussi de la sémiotique et de l'ethnométhodologie, approches disciplinaires dans lesquelles se place le troisième et dernier article de cette partie. Intitulé " Une linguistique au-delà de l'humain? Les vertus heuristiques de la sémiosis inter-espèce", le texte de Chloé Mondémé pose l'hypothèse d'une communication interspécifique co-construite par ajustements mutuels, en écartant la possibilité exclusive d'un " code supposément partagé » et de capacités « human-like » de certaines espèces, postulats qui avaient amené à conclure trop hâtivement à l'impossibilité d'apprentissage langagier chez les primates.

La dernière partie de ce numéro, "Anthropomorphismes et zoo-anthroponymies ", s'attache aux doubles antispécistes linguistiques et discursifs au sens large: comparaisons interlangues, inter-sons, quand les vocalisations animales et humaines sont confrontées, ou même collection et collectionneuse des discours animaliers in absentia nourrissent les interstices où les animaux en disent long sur ceux qui en parlent. Cette partie s'ouvre sur «De l'animalisation à la neutralisation: fonctionnement des verbes de bruit associés aux animaux », où Irina Kor Chahine propose l'inversion des attendus et une focalisation initiale sur les cris d'animaux 
comme centraux : et si l'animalisation du discours était neutralisée dès lors qu'elle s'applique aux parties du corps, objets et autres? Et si l'on partait de l'animal pour ne pas ajouter de charge émotionnelle? Les verba sonandi animaliers regorgent et se séparent entre métaphores neutralisées, non expressives, et constructions humaines où l'animalité nourrit l'expressivité. Loin des méthodes universitaires conventionnelles, "Les affichettes d'animaux perdus. Discursivité, agentivité, anthroponymie » est un dialogue entre Elsa Eskenazi et Marie-Anne Paveau qui propose une approche audacieuse des affichettes d'animaux perdus. À la fois récit de la collection, dans sa constitution tout comme de sa caractérisation, et placement de cette collection en tant que située dans l'espace de la collectionneuse, la seconde partie analyse l'objetaffichette dans sa matérialité (en tant que biographie d'objet), dans sa discursivité (en tant qu'objet discursif graphique) et dans le rapport entre l'humain et l'animal qui se joue dans ses textes et formules. Dans le dernier article de ce volume, "Étude contrastive français-chinois des stéréotypes dans la zoo-anthroponymie ", Lian Chen dresse une typologie des images animalières en chinois et en français, ainsi que de leur caractérisation : le serpent, négatif dans les cultures imprégnées de christianisme, est au contraire l'un des symboles positifs du zodiaque chinois; dans les deux langues existe l'expression « fort comme un bœuf ». Mais d'où viennent les spécismes apparents dans les deux visions stéréotypiques et idiomatiques? L'auteure apporte un début de réponse assorti d'un corpus d'exemples fourni afin de voir si les références aux animaux sont essentiellement négatives ou positives dans ces deux cultures.

Ces articles qui posent les jalons d'une réflexion politique et linguistique sur la faculté langagière animale, au-delà des catégorisations humain/animal, s'inscrivent dans un vaste mouvement actuel qui travaille à modifier des représentations ancrées sur une spécificité humaine. L'analyse du discours, et plus largement la linguistique et la sémiotique, peuvent offrir un lieu de dialogue et de convergence entre les différents travaux dans le domaine de la communication animale, un espace où pourra s'opérer ce "défigement représentationnel» et la redéfinition d'un langage interspécifique incluant l'univers végétal et l'ensemble des actants non humains.

\section{BIBLIOGRAPHIE}

Blog de référence du projet de numéro : ANIMALINGUA. Discours, animalité et humanité, https:// realista.hypotheses.org/.

Baratay, Éric, 2017, Biographies animales. Des vies retrouvées, Paris, Seuil.

Benhaïm, André et Simon, Anne (dir.), 2017, « Zoopoétique. Des animaux en littérature moderne de langue française ", Revue des Sciences Humaines, $\mathrm{n}^{\circ} 328$.

Benveniste, Émile, [1952] 1966, « Communication animale et langage humain », dans Problèmes de linguistique générale 1, Paris, Gallimard, p. 56-62. 
Berger, John, [1995] 2010, « Pourquoi regarder les animaux? », trad. K. Berger Andreadakis, dans J. Berger, Au regard du regard, Paris, L'Arche, p. 7-35, repris dans H.-S. Afeissa et J.-B. Jeangène Vilmer (dir.), Philosophie animale. Différence, responsabilité et communauté, Paris, Vrin, p. 29-54.

Bertrand, Denis et Costantini, Michel, 2017, « La parole aux animaux. Conditions d'extension de l'énonciation », Journée d'étude du GASP8, 27 janvier 2017, Université Paris 8.

Calarco, Matthew, 2010, « Nul ne sait où commence ni où finit le visage. L'humanisme et la question de l'animal », trad. d'un texte inédit par H.-S. Afeissa, dans H.-S. Afeissa et J.-B. Jeangène Vilmer (dir.), Philosophie animale. Différence, responsabilité et communauté, Paris, Vrin, p. 84-124.

David, Bruno (dir.), 2019, Manifeste du muséum. Humains et autres animaux, Paris, Coédition Reliefs, Muséum national d'Histoire naturelle.

Depenau, Mathieu, 2009, « L'animal moral », Le Portique, n²3-24, http://

journals.openedition.org/leportique/2445, consulté le 20 juin 2020.

Derrida, Jacques, 1989, « "Il faut bien manger” ou le calcul du sujet », entretien avec Jean-Luc Nancy, Cahiers confrontation, $\mathrm{n}^{\circ} 20$, p. 91-114.

Derrida, Jacques, 1994, Force de la loi. Le « fondement mystique de l'autorité », Paris, Galilée.

Derrida, Jacques, 2006, L'animal que donc je suis, Paris, Galilée.

Descola, Philippe, 2006, Par-delà nature et culture, Paris, Seuil.

Descola, Philippe, 2011, L'écologie des autres. L'anthropologie et la question de la nature, Paris, Éditions Quae.

Descola, Philippe, 2014, La composition des mondes, Paris, Champs Flammarion.

Descola, Philippe, 2017, « La forêt des signes ", préface à Eduardo Kohn, Comment pensent les forêts, trad. G. Delaplace, Paris, Zones sensibles, p. 11-17.

De Waal, Frans, 1996, Le Bon Singe : les bases naturelles de la morale, Paris, Bayard.

De Waal, Frans, 2001, Quand les singes prennent le thé : de la culture animale, trad. J.-P. Mourlon, Paris, Fayard.

De Waal, Frans, 2010, L'Âge de l'empathie : leçons de nature pour une société plus apaisée ?, trad. M.-F. de Paloméra, Paris, Éditions Les Liens qui libèrent.

De Waal, Frans, 2016, Sommes-nous trop « bêtes » pour comprendre l'intelligence des animaux ?, trad. L. Chemla et P. Chemla, Paris, Les liens qui libèrent.

Fontenay, Élisabeth de, 1998, Le silence des bêtes : la philosophie à l'épreuve de l'animalité, Paris, Fayard.

Fontenay, Élisabeth de, 2008, Sans offenser le genre humain : réflexions sur la cause animale, Paris, Albin Michel.

Goudet, Laura, Paveau, Marie-Anne et Ruchon, Catherine, 2017, « Analyse du discours animal. Une nouvelle perspective pour les sciences du langage », intervention au séminaire de recherche pluridisciplinaire Critiques sociales du langage, Université Paris Descartes, 28 avril.

Harley, Trevor A., 2017, Talking the talk: Language, psychology and science, Londres, Routledge, $2^{\mathrm{e}}$ édition.

Kohn, Fernando, [2013] 2017, Comment pensent les forêts. L'anthropologie au-delà de l'humain, trad. Grégory Delaplace, Paris, Zones sensibles. 
Latour, Bruno, 1991, Nous n'avons jamais été modernes. Essai d'anthropologie symétrique, Paris, La Découverte.

Latour, Bruno, 1995, « Note sur certains objets chevelus », Nouvelle revue d'ethnopsychiatrie, $\mathrm{n}^{\circ} 27$, p. 21-36.

Latour, Bruno, 1999, Politiques de la nature. Comment faire entrer les sciences en démocratie, Paris, La Découverte.

Lecointre, Guillaume, 2013, « Classer les espèces : une question cruciale de la biologie », dans J.J. Kupiec (dir.), La Vie, et Alors ?, Paris, Belin, p. 283-317.

Lestel, Dominique, 2001, Les origines animales de la culture, Paris, Flammarion.

Lestel, Dominique, 2010, L'animal est l'avenir de l'homme, Paris, Fayard.

Marrone, Gianfranco, 2018, «Énonciation animale : Franz Kafka, Primo Levi, le singe et la poule », Actes du Colloque La parole aux animaux. Conditions d'extension de l'énonciation, Université Paris 8Vincennes-Saint-Denis, 27 janvier 2017, Fabula, [En ligne], http://www.fabula.org/colloques/ document5364.php, consulté le 23 juin 2020.

Mondémé, Chloé, 2018, « Comment parle-t-on aux animaux ? Formes et effets pragmatiques de l'adresse aux animaux de compagnie », Langage et société, n 163, p. 77-99.

Nurock, Vanessa, 2008, « Les animaux sont-ils des êtres humains sympathiques ? Perspectives cognitives sur la question d'une "morale animale" ", Revue du MAUSS, n 31, p. 397-410.

Paveau, Marie-Anne, 2012a, « Réalité et discursivité. D’autres dimensions pour la théorie du discours ", Semen, $n^{\circ}$ 34, [En ligne], https://doi.org/10.4000/semen.9748, consulté le 20 juin 2020.

Paveau, Marie-Anne 2012b, « Ce que disent les objets. Sens, affordance, cognition », dans Synergies Pays de la baltique, no 9, revue du GERFLINT, p. 53-65, [En ligne], http://ressourcescla.univ-fcomte.fr/gerflint/Baltique9/baltique9.html, consulté le 20 juin 2020.

Paveau, Marie-Anne, 2017, L'analyse du discours numérique. Dictionnaire des formes et des pratiques, Paris, Hermann.

Pepperberg, Irene M., 2006, « Cognitive and communicative abilities of Grey parrots », Applied Animal Behaviour Science, vol. 100, $\mathrm{n}^{\circ}$ 1, p. 77-86.

Pepperberg, Irene M., 2009, Alex \& Me: How A Scientist And A Parrot Discovered A Hidden World of Animal Intelligence-And Formed A Deep Bond In The Process, New York, Harper.

Pilley, John W. et Reid Allison K., 2011, « Border collie comprehends object names as verbal referents », Behavioural Processes, vol. 86, nº 2, p. 184-195.

Premack, David et Woodruff, Guy, 1978, « Does the chimpanzee have a theory of mind ?», Behavioral and Brain Sciences 1, p. 515-526.

Premack, David et Premack, Ann, [1983] 1984, L'esprit de Sarah, trad. Y. Baudry, Paris, Fayard. Proust, Joëlle, 1997, Comment l'esprit vient aux bêtes. Essai sur la représentation, Paris, Gallimard.

Proust, Joëlle, 2000, « L'animal intentionnel », Terrain, n 34, [En ligne], https://doi.org/10.4000/ terrain.944, consulté le 20 juin 2020.

Proust, Joëlle, 2010, Les animaux pensent-ils ?, Paris, Bayard Culture.

Rabatel, Alain, 2017, « Du “point de vue” animal », Le discours et la langue, vol. 9, n 2, p. 145-157.

Rivera, Annamaria, 2015, « Sexisme, racisme, spécisme : notes introductives à une dialectique complexe », dans Mélanges en l'honneur de Mondher Kilani, Bangkok, BSN Press, p. 133-144. 
Rubi, Serge, 1992, «Quelques aspects de la communication verbale Homme-Chat », Tranel, $\mathrm{n}^{\circ}$ 18, p. 231-243.

Ruchon, Catherine, 2017, « De l'anthropodéni aux anthropomorphèmes, les frontières linguistiques de l'humanité », Animalingua. Discours, animalité et humanité [carnet de recherche], 6 janvier 2017, https://realista.hypotheses.org/1372, consulté le 20 juin 2020.

Ruchon, Catherine, 2018, « Lexique, catégorisation et représentation : les reformulations métalinguistiques dans le discours animaliste », Les Carnets du Cediscor, $\mathrm{n}^{\circ}$ 14, https://doi.org/ 10.4000/cediscor.1226, consulté le 20 juin 2020.

Schaeffer, Jean-Marie, 2007, La fin de l'exception humaine, Paris, Gallimard.

Simon, Anne, 2015, « French Literary Studies and The Animal Question: A Contemporary Perspective ", dans L. Mackenzie et S. Posthumus (dir.), Through the Cultural Prism: Thinking about Animals from a French and Francophone Perpective, Michigan Press, parution en français sur le site Épistémocrique, http://epistemocritique.org/les-etudes-litteraires-francaises-et-la-question-delanimalite-xxe-xxie-siecles-bilan-et-perspectives-en-zoopoetique/, consulté le 20 juin 2020.

Uexküll, Jakob von, [1934] 1965, Mondes animaux et monde humain suivi de Théorie de la signification, trad. P. Muller, Paris, Denoël.

Vauclair, Jacques, 1995, L'intelligence de l'animal, Paris, Seuil.

\section{NOTES}

1. Pour une discussion sur le classement des espèces, voir par exemple la troisième édition du Manifeste du Muséum de Bruno David (2019) ou les travaux du zoologiste Guillaume Lecointre (2013 notamment) sur la classification par emboîtement (qui fait de l'humaine un primate), et sur l'indétermination lexicographique de homme/humain et animal, entre hyponyme et hyperonyme, voir Ruchon (2018).

\section{AUTEURS}

\section{LAURA GOUDET}

Université de Rouen, ERIAC (ER 4705)

\section{MARIE-ANNE PAVEAU}

Université Sorbonne Paris Nord, Pléiade (ER 7338)

\section{CATHERINE RUCHON}

Université Montpellier 3, Pléiade (ER 7338) 